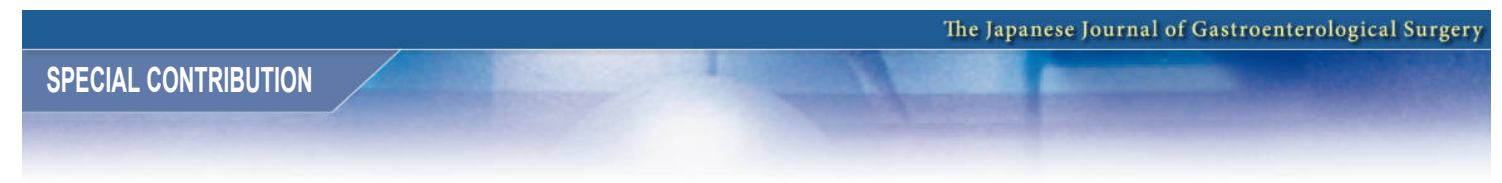

\title{
How Readability Can Improve Your Manuscript
}

\author{
Takako Kojima ${ }^{1)}$ and J. Patrick Barron ${ }^{2)}$ \\ ${ }^{1)}$ Department of International Medical Communications, Tokyo Medical University \\ 2) Tokyo Medical University
}

The ICMJE Recommendations are guidelines that the vast majority of international journals follow. However, the ICMJE states that it is essential for authors to study the Instructions to Authors of the target journal for submission, in addition to its Recommendations. Please note that the ICMJE Recommendations should be used for basic, general knowledge about medical writing, and authors should additionally consult the Instructions to Authors for the target journal when preparing manuscripts for submission ${ }^{1)}$.

The quality of your research and the quality of your manuscript are different things. If your manuscript lacks readability and contains simple writing mistakes, it will probably decrease your chance of being accepted. Although every author will have a different style in writing, scientific writing, in particular, must be precise and unambiguous to be effective ${ }^{2)}$. Here are some tips on how to enhance the overall quality of your manuscript.

\section{Readability}

When you prepare your manuscript, it is advisable to put yourself in the shoes of the referee who will be reviewing it. Reviewing manuscripts is (almost universally) unpaid, volunteer work, and part of a "culture of service" which is integral to the profession ${ }^{3}$. Since most referees review manuscripts after a full day of work, often late at night when they are tired, if the manuscript they are reviewing includes many easily-avoidable simple mistakes, or if it is written using long, unclear, complicated sentences the referee may become seriously less motivated to read your manuscript, which will then lead to a negative impression of your research. This is one particular reason readability becomes extremely important when preparing a manuscript. Readability is defined as "the quality of written language that makes it easy to read and understand"4). Nobody, including the referee, wants to read pages of turgid, repetitious sentences replete with basic writing mistakes. Elementary mistakes in spelling, punctuation, spacing, and grammar give a very poor impression. Therefore it is important to remember to use sharp, accurate sentences. Difficult vocabulary and long sentences diminish the manuscript readability.

\section{Avoiding Simple Mistakes}

When preparing manuscripts for submission, it is essential for authors to proofread their manuscripts carefully from the point of view of a well-educated non-specialist reader, so that any simple mistakes or difficult points can be avoided ${ }^{5)}$. The American Medical Association (AMA) Manual of Style provides useful information on how to avoid common grammatical and writing errors and gives examples of correct and preferred usage of words. One example is the usage of the words 'management' and 'treatment'. The AMA states that it is generally better to say that cases are managed and patients are cared for or treated, although there are exceptions, such as in the case of "the clinical management of the patient with AIDS"5).

\section{Putting Referees' Comments to Use}

Many similarities can be found in some of the referees' comments about style. Some common criticisms from referees include, manuscripts being too long, too many keywords, unclear Discussion, repetitious information, generally unclear and poorly written, inappropriate structure, and topics that do not attract the interest of readers. Authors can benefit from such comments in their future manuscript writing. For example, there is a tendency for 
authors to begin the Discussion section with background information, or repeat information that is already mentioned in the Introduction. This makes it difficult for the reader to remember exactly what the objective of the study was. Therefore, it is more effective to begin with a sentence such as, "We set out to determine whether ..." to clearly point out and remind the readers of the original goal of the study.

\section{Frontloading Sentences}

Frontloading means that the most important information is mentioned at the beginning of the sentence, rather than at the end, which is a more typical structure found in written Japanese. When a sentence is frontloaded, the reader can quickly assess the information for significant points, and therefore it will attract the attention of the reader and make it more readily understandable. For example, if you compare the following 2 sentences, "The patient came to the hospital and because he had a high fever he was admitted" and "The patient was admitted because of a high fever", the latter sentence is clearer to understand as it gives the conclusion "the patient was admitted" at the beginning, and tells the reader what happened first, followed by the reason.

\section{Summary}

When preparing manuscripts for submission, it is important that much time is spent revising as every good piece of writing requires many revisions. Using a spell checker to check for simple spelling mistakes; not neglecting punctuation; avoiding usage of the same words; structuring clear, strong, and organized sentences; and careful proofreading, are essential in preparing and improving the quality of a manuscript.

\section{References}

1) Kojima T, Barroga E. Preparing manuscripts in accordance with the 'Recommendations for the Conduct, Reporting, Editing and Publication of Scholarly Work in Medical Journals'. 日本消化器外科学会雑誌. 2014;47(3):212-3.

2) American Chemical Society. The ACS style guide: effective communication of scientific information. 3rd ed. New York: Oxford University Press; 2006. Chapter 4, Writing style and word usage; p. 41.

3) Lovejoy, TI, Revenson TA, France CR. Reviewing manuscripts for peer-review journals: a primer for novice and seasoned reviewers. Ann Behave Med. 2011;42:1-13.

4) Webster online dictionary [Internet]. Pennsylvania: Interapple, Inc; [cited 2014 Nov 25]. Available from: http://www.websterdictionary.org/.

5) American Medical Association. AMA manual of style: a guide for authors and editors. 10th ed. New York: Oxford University Press; 2007. Chapter 11, Correct and preferred usage; p. 381, 398. 